\title{
Long-term Oncologic Outcome of Postoperative Complications After Colorectal Cancer Surgery
}

\author{
Chang Kyu Oh, Jung Wook Huh, You Jin Lee, Moon Suk Choi, Dae Hee Pyo, Sung Chul Lee, Seong Mun Park, \\ Jung Kyong Shin, Yoon Ah Park, Yong Beom Cho, Seong Hyeon Yun, Hee Cheol Kim, Woo Yong Lee \\ Department of Surgery, Samsung Medical Center, Sungkyunkwan University School of Medicine, Seoul, Korea
}

Purpose: The impact of postoperative complications on long-term oncologic outcome after radical colorectal cancer surgery is controversial. The aim of this study was to examine the risk factors and oncologic outcomes of surgery-related postoperative complication groups.

Methods: From January 2010 to December 2010, 310 patients experienced surgery-related postoperative complications after radical colorectal cancer surgery. These stage I-III patients were classified into 2 subgroups, minor (grades I, II) and major (grades III, IV) complication groups, according to extended Clavien-Dindo classification system criteria. Clinicopathologic differences between the 2 groups were analyzed to identify risk factors for major complications. The diseasefree survival rates of surgery-related postoperative complication groups were also compared.

Results: Minor and major complication groups were stratified with 194 patients (62.6\%) and 116 patients (37.4\%), respectively. The risk factors influencing the major complication group were pathologic $\mathrm{N}$ category and operative method. The prognostic factors associated with disease-free survival were preoperative perforation, perineural invasion, tumor budding, and receiving neoadjuvant therapy. With a median follow-up period of 72.2 months, the 5 -year disease-free survival rates were $84.4 \%$ in the minor group and $78.5 \%$ in the major group, but there was no statistical significance between the minor and major groups $(\mathrm{P}=0.392)$.

Conclusion: Advanced cancer and open surgery were identified as risk factors for increased surgery-related major complications after radical colorectal cancer surgery. However, severity of postoperative complications did not affect disease-free survival from colorectal cancer.

Keywords: Postoperative complications; Colorectal cancer surgery; Risk factor; Recurrence

\section{INTRODUCTION}

Colorectal cancer is the third most commonly occurring cancer in males and the second most commonly occurring cancer in females. Over 1.8 million new cases and 881,000 deaths occurred in

Received: Aug 30, 2019 - Revised: Oct 14, 2019 - Accepted: Oct 15, 2019 Correspondence to: Jung Wook Huh, M.D.

Division of Colorectal Surgery, Department of Surgery, Samsung Medical Center, Sungkyunkwan University School of Medicine, 81 Irwon-ro,

Gangnam-gu, Seoul 06351, Korea

Tel: +82-2-3410-1826, Fax: +82-2-3410-6980

E-mail: jungwook.huh@gmail.com

ORCID: https://orcid.org/0000-0002-4485-0184

(C) 2020 The Korean Society of Coloproctology

This is an open-access article distributed under the terms of the Creative Commons Attribution NonCommercial License (https://creativecommons.org/licenses/by-nc/4.0) which permits unrestricted noncommercial use, distribution, and reproduction in any medium, provided the original work is properly cited.
2018 [1]. Surgical resection is the main treatment of choice for patients with stages I-III colorectal cancer [2]. Despite advancements in surgical methods, postoperative complications are observed in up to $35 \%$ of patients who undergo radical colorectal cancer surgery [3-8]. The impact of postoperative complications that affect long-term oncologic outcomes after radical colorectal surgery is controversial [8-12].

Some authors have shown that injuries associated with surgery stimulate tumor growth and dissemination [13-15]. Other researchers have shown that postoperative complications affect the immunological response, which improves undetected tumor viability and increases disease recurrence $[16,17]$. In most studies, researchers analyzed all complications after curative colorectal cancer surgery, whether related to the surgery or not $[9,12,18$, 19]. The elimination of complications not related to surgery, i.e., those beyond the surgeon's control, would provide meaningful 
analysis in identification of surgical factors.

The aim of this study was to examine the relationship between minor and major surgery-related complication subgroups according to the Clavien-Dindo (CD) classification system. The influence of surgery-related postoperative complications on diseasefree survival between these 2 subgroups was compared.

\section{METHODS}

\section{Patients}

We retrospectively analyzed 1,149 patients who underwent radical surgery for stages I-III colorectal cancer at Samsung Medical Center from January 2010 to December 2010. The exclusion criteria were (1) recurrent colorectal cancer, (2) metachronous cancer, (3) hereditary colorectal cancer, and (4) radical surgery not performed (patient underwent local excision and polypectomy). Among 1,149 patients, 334 experienced postoperative complications within 30 days after colorectal radical surgery. Among these 334 patients, 24 experienced complications not related to surgery. This study was reviewed and approved by the Institutional Review Board at Samsung Medical Center (IRB No. 2019-08-056001), and informed consent was waived.

All patients underwent curative surgery performed by 6 welltrained colorectal surgeons. Cancer staging followed the 7th American Joint Committee on Cancer (AJCC) TNM classification guidelines [20]. According to radiologic evaluation, patients with locally advanced (radiological T3/T4 or N positive) rectal cancer located within $10 \mathrm{~cm}$ of the anal verge received preoperative therapy with a 5-fluorouracil-based chemotherapy regimen or underwent radiotherapy of the whole pelvis at a dose of 40.4-50.4 Gy.

Five-fluorouracil-based chemo-regimen was preferentially considered as adjuvant chemotherapy for patients with T3, T4, or node-positive disease after surgical resection. This decision was based on patient health status, compliance, and physician preference. Patients were followed up at 3-month intervals for the first 2 years, at 6-month intervals for the next 3 years, and yearly thereafter. Follow-up surveillance examinations included clinical history, physical examination, measurement of serum carcinoembryonic antigen level, chest X-ray, and chest and abdominopelvic computed tomography scan. Additional pelvic magnetic resonance imaging and positron emission tomography scans were performed every 6 to 12 months depending on patient status. Colonoscopy was performed 1 year after surgery and then every 2 years [20].

The 310 patients were retrospectively placed into 5 categories according to the extended CD classification system for surgical complications published by the Japan Clinical Oncology Group in 2016 [21]. The CD classification system published in 2004 provided for simple categorization of postoperative complications [22]. CD classification, along with the extended CD classification system, was established to standardize adverse event terms and provide more detailed grading guidelines in clinical practice [21].
Postoperative morbidity was defined as a complication occurring during the hospital stay or within 30 days after surgery. Patients were assigned to 2 groups according to $\mathrm{CD}$ classification: minor complication group including $\mathrm{CD}$ grades I to II and major complication group including CD grades III to IV.

\section{Statistical analysis}

Data were analyzed using IBM SPSS Statistics ver. 23.0 (IBM Corp., Armonk, NY, USA). Clinicopathologic features of the 2 groups were analyzed using chi-square test or t-test. Continuous variables were recorded as the median and minimum to maximum ranges. Variables with P-value $<0.05$ were considered statistically significant in univariate analysis. Using the variables identified as statistically significant by univariate analysis, multivariate analysis was carried out using the logistic regression model and Cox regression method. Disease-free survival rates were estimated using the Kaplan-Meier method, and differences between curves were compared using the log-rank test.

\section{RESULTS}

A total of 1,149 patients with stage I-III colorectal cancer underwent curative radical surgery in 2010 . Of these patients, $334 \mathrm{pa}-$ tients $(29.1 \%)$ had postoperative complications; 24 patients $(2.1 \%)$ who experienced complications unrelated to surgery were excluded. The remaining 310 patients (27.0\%) were categorized into 1 of 5 grades according to CD classification: (1) 52 patients (4.5\%) were CD grade I; (2) 142 patients (12.4\%) were CD grade II; (3) 78 patients $(6.8 \%)$ were $\mathrm{CD}$ grade IIIa; 35 patients $(3.0 \%)$ were $\mathrm{CD}$ grade IIIb; and (4) 3 patients $(0.3 \%)$ were CD grade IVa. Severe complications such as $\mathrm{CD}$ grade IVb and mortality were absent. The type and frequency of complications are shown in Table 1. The most common type of complication related to surgery was wound complication (31.6\%); postoperative ileus (19.7\%) was the second most common complication. Anastomosis leakage occurred in $11.9 \%$ of cases and was the second most common complication $(20.7 \%)$ in the major complication group. Most complications unrelated to surgery were in the minor complication group (91.7\%).

Patients were divided into 2 subgroups, a minor complication group (CD grade I/II) and a major complication group (CD grade III/IV) containing 194 and 116 patients, respectively. Comparisons of clinicopathologic features of patients according to complication groups (CD grade I/II vs. III/IV) are shown in Table 2. Preoperative obstruction, operative method, length of hospital stay, pathologic $\mathrm{N}$ category, and tumor size differed significantly between the 2 groups.

Risk factors influencing the major complication group are shown in Table 3. Univariate logistic regression indicated that preoperative obstruction, operative method, pathologic $\mathrm{N}$ category, and neoadjuvant therapy were risk factors. On multivariate analysis, operative method, and pathologic $\mathrm{N}$ category were inde- 
Table 1. Type and frequency of complication

\begin{tabular}{|c|c|c|c|c|c|c|}
\hline \multirow{2}{*}{ Type } & \multirow{2}{*}{ Total } & \multicolumn{5}{|c|}{ Clavien-Dindo classification } \\
\hline & & I & $\|$ & IIla & Illb & IV \\
\hline Surgery-related & & \multicolumn{2}{|c|}{ Minor complication 194 (62.6) } & \multicolumn{3}{|c|}{ Major complication 116 (37.4) } \\
\hline lleus & $61(19.7)$ & $11(3.5)$ & $40(12.9)$ & $7(2.3)$ & $2(0.6)$ & $1(0.3)$ \\
\hline Urinary retention & $58(18.7)$ & $13(4.2)$ & $45(14.5)$ & - & - & - \\
\hline Postoperative bleeding & $27(8.7)$ & $7(2.3)$ & $16(5.2)$ & - & $3(1.0)$ & $1(0.3)$ \\
\hline Chylous ascites & $13(4.2)$ & $1(0.3)$ & $12(3.9)$ & - & - & - \\
\hline Intraabdominal fluid collection & $12(3.9)$ & - & $4(1.3)$ & $7(2.3)$ & $1(0.3)$ & - \\
\hline Rectovaginal fistula & $2(0.6)$ & - & - & - & $2(0.6)$ & - \\
\hline Bowel perforation & $2(0.6)$ & - & - & - & $2(0.6)$ & - \\
\hline Urinary dysfunction & $4(16.7)$ & - & $3(12.5)$ & - & $1(4.2)$ & - \\
\hline Neurologic complication & $3(12.5)$ & $1(4.2)$ & $2(8.3)$ & - & - & - \\
\hline Cardiac complication & $2(8.3)$ & - & $2(8.3)$ & - & - & - \\
\hline Other & $2(8.3)$ & - & $2(8.3)$ & - & - & - \\
\hline
\end{tabular}

Values are presented as number (\%).

pendent risk factors for the major complication group.

In the median follow-up of 72.2 months (range, 0.2 to 113.6 months), the 5-year disease-free survival rate of this cohort was $81.5 \%$. The 5-year disease-free survival rates of the minor complication group (CD grades I and II) and the major complication group (CD grades III and IV) were $84.4 \%$ and $78.5 \%$, respectively. There was no statistically significant difference between minor and major complication groups $(\mathrm{P}=0.392)$ (Fig. 1). The survival curves according to pathologic TNM stage differed significantly. The 5-year disease-free survival rates of patients with pStage 0 , pStage I, pStage II, and pStage III were 95.5\%, 94.1\%, 84.2\%, and $69.8 \%$, respectively $(\mathrm{P}<0.001)$ (Fig. 2).

Univariate analysis indicated that the prognostic factors associated with disease-free survival were preoperative perforation, emergency surgery, transfusion during operation, pathologic $\mathrm{T}$ category, pathologic N category, lymphovascular invasion (LVI), perineural invasion (PNI), tumor budding, receiving neoadjuvant therapy, and adjuvant therapy. On multivariate analysis, preoperative perforation, PNI, tumor budding, and receiving neoadjuvant therapy were independent prognostic factors for disease-free survival (Table 4).

\section{DISCUSSION}

In this study, 334 patients (29.1\%) with postoperative complications after radical colorectal cancer surgery were investigated.
Among these, 310 patients (27.0\%) experienced surgery-related complications. The surgery-related complication cohort was classified into 2 subgroups, minor and major complication groups, encompassing 194 patients (62.6\%) and 116 patients (37.4\%), respectively. These complication rates are consistent with those of previous studies [3-8, 19, 23, 24]. Risk factors for major surgeryrelated complications were analyzed. There were differences in the operative method and pathologic $\mathrm{N}$ category between minor and major complication groups. Pathologic N category was associated with severity of cancer stage; pathologic $\mathrm{N}$ category positive refers to stage III colorectal cancer or higher according to the 7 th AJCC TNM classification. Previous studies have shown a relationship between higher cancer stage and an increased incidence of postoperative complications $[8,25,26]$.

Open surgery is considered a risk factor for increased surgeryrelated major complications. Laparoscopic surgery has advantages over open surgery with no difference in oncologic outcome; advantages include less blood loss, shorter length of wound, faster recovery of bowel function, and shorter hospital stay [3-5]. The laparoscopic approach reduces wound incision and decreases the possibility of wound complications such as seroma, infection, and the need for resuturing under local anesthesia. These complications are categorized as major complications by the extended CD classification system [21].

The impact of postoperative complications that affect oncologic long-term outcomes in colorectal cancer remains controversial. 
Table 2. Clinicopathologic features of surgery-related complications cohort $(n=310)$

\begin{tabular}{|c|c|c|c|}
\hline Variable & $\begin{array}{l}\text { CD grades I, II } \\
\quad(n=194)\end{array}$ & $\begin{array}{l}\text { CD grades III, IV } \\
\quad(n=116)\end{array}$ & P-value \\
\hline \multicolumn{4}{|l|}{ Preoperative features } \\
\hline Age (yr) & 60.5 (33-83) & 59 (32-85) & 0.545 \\
\hline \multicolumn{4}{|l|}{ Sex } \\
\hline Female & $67(65.5)$ & $38(32.8)$ & 0.749 \\
\hline Male & $127(65.5)$ & $78(67.2)$ & \\
\hline \multicolumn{4}{|l|}{ ASA PS classification } \\
\hline I, II & $176(95.7)$ & $102(94.4)^{\mathrm{a}}$ & 0.641 \\
\hline III, IV & $8(4.3)$ & $6(5.6)$ & \\
\hline Body mass index $\left(\mathrm{kg} / \mathrm{m}^{2}\right)$ & $\begin{array}{c}23.78 \\
(15.12-35.34)\end{array}$ & $\begin{array}{c}23.46 \\
(16.45-30.52)\end{array}$ & 0.415 \\
\hline Preoperative CEA level (ng/mL) & $\begin{array}{c}1.83 \\
(0.5-126.37)\end{array}$ & $\begin{array}{c}2.23 \\
(0.5-125.70)^{b}\end{array}$ & 0.465 \\
\hline \multicolumn{4}{|l|}{ Underlying disease } \\
\hline Hypertension & $63(32.5)$ & $42(36.2)$ & 0.502 \\
\hline Diabetes mellitus & 38 (19.6) & $21(18.1)$ & 0.747 \\
\hline Heart disease & $14(7.2)$ & $7(6.0)$ & 0.689 \\
\hline Cerebrovascular disease & $8(4.1)$ & $1(0.9)$ & 0.980 \\
\hline Pulmonary disease & $12(6.2)$ & $8(6.9)$ & 0.805 \\
\hline Previous abdominal surgery & $57(29.4)$ & 35 (30.2) & 0.883 \\
\hline \multicolumn{4}{|l|}{ Preoperative treatment } \\
\hline Chemotherapy & $1(0.5)$ & $1(0.7)$ & 0.130 \\
\hline Radiotherapy & $0(0)$ & $0(0)$ & \\
\hline Chemoradiotherapy & $28(14.4)$ & $27(23.3)$ & \\
\hline Preoperative stenting & $2(1.0)$ & $3(2.6)$ & 0.293 \\
\hline Obstruction & $19(9.8)$ & $23(19.8)$ & 0.012 \\
\hline Perforation & $6(3.1)$ & $9(7.8)$ & 0.064 \\
\hline \multicolumn{4}{|l|}{ Intraoperative features } \\
\hline \multicolumn{4}{|l|}{ Type of operation } \\
\hline Right hemicolectomy & $57(29.4)$ & 25 (21.6) & 0.211 \\
\hline Left hemicolectomy & $6(3.1)$ & $7(6.0)$ & \\
\hline Anterior resection & $32(16.5)$ & $17(14.7)$ & \\
\hline Low anterior resection & $92(47.4)$ & $56(48.3)$ & \\
\hline Other & $7(3.6)$ & $11(9.5)$ & \\
\hline \multicolumn{4}{|l|}{ Level of anastomosis } \\
\hline Colon & $111(57.2)$ & $60(51.7)$ & 0.347 \\
\hline Rectum & $83(42.8)$ & $56(48.3)$ & \\
\hline \multicolumn{4}{|l|}{ Operative method } \\
\hline Open surgery & $94(48.5)$ & $82(70.7)$ & $<0.001$ \\
\hline Minimally invasive surgery & $100(51.5)$ & $34(29.3)$ & \\
\hline Emergency surgery & $7(3.6)$ & $6(5.2)$ & 0.506 \\
\hline Diverting stoma & $37(19.1)$ & $20(17.2)$ & 0.687 \\
\hline Operative time (min) & $160(43-492)$ & $157(64-490)$ & 0.827 \\
\hline Transfusion during operation & $6(3.1)$ & $7(6.0)$ & 0.211 \\
\hline
\end{tabular}

(Continued to the next)
Table 2. Continued

\begin{tabular}{|c|c|c|c|}
\hline Variable & $\begin{array}{l}\text { CD grades I, II } \\
\quad(n=194)\end{array}$ & $\begin{array}{c}\text { CD grades III, IV } \\
(n=116)\end{array}$ & P-value \\
\hline \multicolumn{4}{|l|}{ Postoperative features } \\
\hline Length of hospital stay & $12(7-44)$ & $17(8-85)$ & $<0.001$ \\
\hline \multicolumn{4}{|l|}{ Pathologic T category } \\
\hline $0,1,2$ & $83(42.8)$ & $37(31.9)$ & 0.057 \\
\hline 3,4 & $111(57.2)$ & $79(68.1)$ & \\
\hline \multicolumn{4}{|l|}{ Pathologic N category } \\
\hline Negative & $122(62.9)$ & $57(49.1)$ & 0.018 \\
\hline Positive & $72(37.1)$ & $59(50.9)$ & \\
\hline \multicolumn{4}{|l|}{ Lymphovascular invasion } \\
\hline Negative & $127(65.5)$ & $70(60.3)$ & 0.365 \\
\hline Positive & $67(34.5)$ & $46(39.7)$ & \\
\hline \multicolumn{4}{|l|}{ Perineural invasion } \\
\hline Negative & 173 (89.2) & $102(87.9)$ & 0.738 \\
\hline Positive & $21(10.8)$ & $14(12.1)$ & \\
\hline \multicolumn{4}{|l|}{ Tumor budding } \\
\hline Negative & $124(63.9)$ & $71(61.2)$ & 0.633 \\
\hline Positive & $70(36.1)$ & $45(38.8)$ & \\
\hline Tumor size (cm) & $3.5(0.1-12)$ & $4.0(0.2-20)$ & 0.021 \\
\hline Harvested lymph nodes & $16(0-58)$ & $17(2-62)$ & 0.173 \\
\hline \multicolumn{4}{|l|}{ Adjuvant therapy } \\
\hline Chemotherapy & $90(46.4)$ & $65(56.0)$ & 0.102 \\
\hline Radiotherapy & $0(0)$ & $1(0.9)$ & \\
\hline Chemoradiotherapy & $16(8.2)$ & $12(10.3)$ & \\
\hline
\end{tabular}

Values are presented as median (range) or number (\%).

ASA PS, American Society of Anesthesiologists physical status; CEA, carcinoembryonic antigen; $\mathrm{CD}$, Clavien-Dindo classification.

${ }^{a}$ Not available (N/A) 18 cases. bN/A 12 cases.

Recent studies have shown that postoperative complications have a negative effect on the oncologic outcome. In particular, infectious complications, surgical intensive care unit admission, and location of anastomosis leakage were associated with negative prognosis $[7,11,12,23,27-29]$. Several studies demonstrated that postoperative complications impact short-term and long-term survival as described above. However, some researchers contend that postoperative complications do not have a significant effect on the oncologic outcome or disease-free survival in colorectal cancer $[8,9,30]$. In our study, advanced cancer stage and operative method were surgery-related risk factors for major complications after radical colorectal cancer surgery. However, the results show no differences in 5-year disease-free survival between minor (84.4\%) and major $(78.5 \%)$ complication groups. These surgeryrelated major complications did not influence disease-free survival $(\mathrm{P}=0.392)$ of colorectal cancer patients.

As described in our study, preoperative perforation, PNI, tumor budding, and receiving neoadjuvant therapy were prognostic factors of disease-free survival in colorectal cancer surgery. These re- 
Table 3. Risk factors influencing Clavien-Dindo grades III and IV in surgery-related complication cohort

\begin{tabular}{|c|c|c|c|c|c|c|}
\hline \multirow{2}{*}{ Variable } & \multicolumn{3}{|c|}{ Univariate analysis } & \multicolumn{3}{|c|}{ Multivariate analysis } \\
\hline & $\mathrm{OR}$ & $95 \% \mathrm{Cl}$ & P-value & $\mathrm{OR}$ & $95 \% \mathrm{Cl}$ & P-value \\
\hline $\operatorname{Age}(>59 / \leq 59 \mathrm{yr})$ & 0.908 & $0.573-1.439$ & 0.682 & & & \\
\hline Sex (male/female) & 1.083 & $0.665-1.764$ & 0.749 & & & \\
\hline ASA PS classification (III, IV/I, II) & 1.294 & $0.437-3.835$ & 0.642 & & & \\
\hline $\mathrm{BMI}\left(>23.6 / \leq 23.6 \mathrm{~kg} / \mathrm{m}^{2}\right)$ & 0.871 & $0.550-1.381$ & 0.558 & & & \\
\hline Preoperative CEA (>5.0/ $\leq 5.0 \mathrm{ng} / \mathrm{mL})$ & 1.310 & $0.708-2.426$ & 0.390 & & & \\
\hline \multicolumn{7}{|l|}{ Underlying disease } \\
\hline Hypertension & 1.180 & $0.728-1.914$ & 0.502 & & & \\
\hline Diabetes mellitus & 0.907 & $0.503-1.638$ & 0.747 & & & \\
\hline Heart disease & 0.826 & $0.323-2.109$ & 0.689 & & & \\
\hline Cerebrovascular disease & 0.202 & $0.025-1.638$ & 0.134 & & & \\
\hline Pulmonary disease & 1.123 & $0.445-2.835$ & 0.805 & & & \\
\hline Preoperative stenting & 2.549 & $0.420-15.484$ & 0.309 & & & \\
\hline Obstruction & 2.278 & $1.180-4.397$ & 0.014 & 1.750 & $0.886-3.457$ & 0.107 \\
\hline Perforation & 2.636 & $0.913-7.606$ & 0.073 & & & \\
\hline Operative method (open/MIS) & 2.566 & $1.573-4.184$ & $<0.001$ & 2.486 & $1.519-4.069$ & $<0.001$ \\
\hline Emergency surgery & 1.457 & $0.478-4.446$ & 0.508 & & & \\
\hline Operative time ( $>160 / \leq 160 \mathrm{~min})$ & 0.973 & $0.614-1.541$ & 0.906 & & & \\
\hline Transfusion during operation & 2.012 & $0.659-6.140$ & 0.219 & & & \\
\hline Pathologic T category $(3,4 / 0,1,2)$ & 1.597 & $0.985-2.588$ & 0.058 & & & \\
\hline Pathologic N category (+/-) & 1.754 & $1.100-2.796$ & 0.018 & 1.661 & $1.031-2.676$ & 0.037 \\
\hline Lymphovascular invasion (+/-) & 1.246 & $0.774-2.004$ & 0.365 & & & \\
\hline Perineural invasion (+/-) & 1.131 & $0.551-2.321$ & 0.738 & & & \\
\hline Tumor budding (+/-) & 1.123 & $0.698-1.805$ & 0.633 & & & \\
\hline Tumor size $(>3.6 / \leq 3.6 \mathrm{~cm})$ & 1.180 & $0.744-1.870$ & 0.481 & & & \\
\hline Neoadjuvant therapy (+/-) & 2.737 & $1.397-5.364$ & 0.003 & 1.519 & $0.808-2.858$ & 0.195 \\
\hline
\end{tabular}

OR, odds ratio; Cl, confidence interval; ASA PS, American Society of Anesthesiologists physical status; BMl, body mass index; CEA, carcinoembryonic antigen; MIS, minimally invasive surgery.

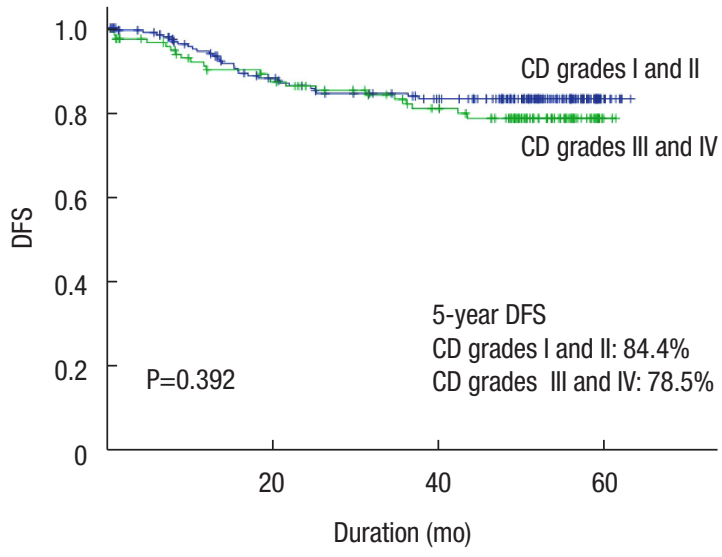

Fig. 1. Disease-free survival (DFS) curves by postoperative complication group of Clavien-Dindo (CD) classification after colorectal cancer surgery.

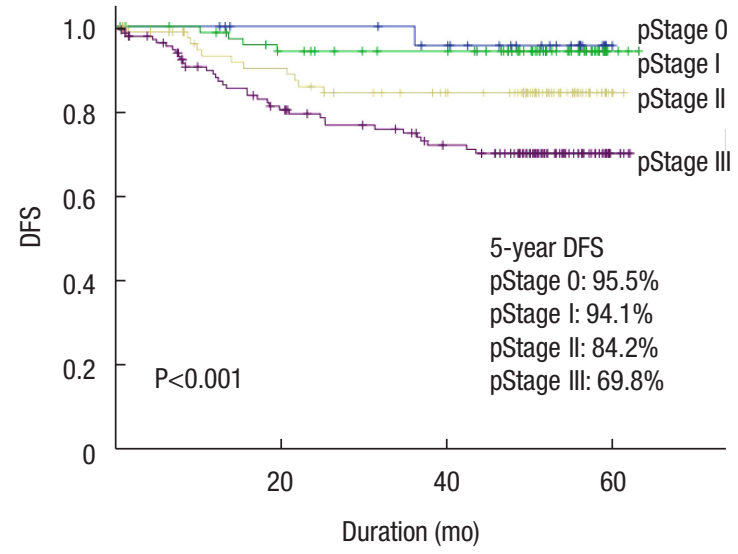

Fig. 2. Disease-free survival (DFS) curves according to pathologic TNM stage (pStage) after colorectal cancer surgery. 


\section{Coloproctology}

Table 4. Prognostic factors influencing disease-free survival in surgery-related complication cohort

\begin{tabular}{|c|c|c|c|c|c|c|}
\hline \multirow{2}{*}{ Pathologic T category $(3,4 / 0,1,2)$} & \multicolumn{3}{|c|}{ Univariate analysis } & \multicolumn{3}{|c|}{ Multivariate analysis } \\
\hline & $\mathrm{OR}$ & $95 \% \mathrm{Cl}$ & P-value & $\mathrm{OR}$ & $95 \% \mathrm{Cl}$ & P-value \\
\hline $\operatorname{Age}(>59 / \leq 59 \mathrm{yr})$ & 0.635 & $0.362-1.114$ & 0.114 & & & \\
\hline Sex (male/female) & 0.583 & $0.336-1.010$ & 0.054 & & & \\
\hline ASA PS classification (III, IV/I, II) & 1.385 & $0.430-4.460$ & 0.585 & & & \\
\hline $\mathrm{BMI}\left(>23.6 / \leq 23.6 \mathrm{~kg} / \mathrm{m}^{2}\right)$ & 0.882 & $0.509-1.527$ & 0.653 & & & \\
\hline Preoperative CEA (>0.5/ $\leq 5.0 \mathrm{ng} / \mathrm{mL})$ & 1.842 & $0.975-3.483$ & 0.060 & & & \\
\hline \multicolumn{7}{|l|}{ Underlying disease } \\
\hline Hypertension & 0.757 & $0.409-1.401$ & 0.376 & & & \\
\hline Diabetes mellitus & 0.464 & $0.184-1.168$ & 0.103 & & & \\
\hline Heart disease & 0.531 & $0.129-2.185$ & 0.381 & & & \\
\hline Cerebrovascular disease & 2.407 & $0.749-7.731$ & 0.140 & & & \\
\hline Pulmonary disease & 0.931 & $0.290-2.988$ & 0.904 & & & \\
\hline Preoperative stenting & 3.012 & $0.731-12.416$ & 0.127 & & & \\
\hline Obstruction & 1.013 & $0.456-2.250$ & 0.974 & & & \\
\hline Perforation & 4.608 & $2.159-9.837$ & $<0.001$ & 4.436 & $2.024-9.722$ & $<0.001$ \\
\hline Operative method (open/MIS) & 1.563 & $0.873-2.797$ & 0.133 & & & \\
\hline Emergency surgery & 3.218 & $1.372-7.549$ & 0.007 & 0.927 & $0.350-2.456$ & 0.879 \\
\hline Operative time ( $>160 / \leq 160 \mathrm{~min})$ & 1.693 & $0.965-2.971$ & 0.067 & & & \\
\hline Transfusion during operation & 2.970 & $1.178-7.486$ & 0.021 & 1.635 & $0.501-5.338$ & 0.416 \\
\hline Pathologic $T$ category $(3,4 / 0,1,2)$ & 3.288 & $1.600-6.7155$ & 0.001 & 1.133 & $0.446-2.880$ & 0.793 \\
\hline Pathologic N category (+/-) & 3.361 & $1.860-6.074$ & $<0.001$ & 1.787 & $0.824-3.876$ & 0.142 \\
\hline Lymphovascular invasion (+/-) & 3.365 & $1.907-5.939$ & $<0.001$ & 1.072 & $0.518-2.217$ & 0.852 \\
\hline Perineural invasion (+/-) & 5.665 & $3.183-10.082$ & $<0.001$ & 3.249 & $1.779-5.934$ & $<0.001$ \\
\hline Tumor budding (+/-) & 4.076 & $2.276-7.300$ & $<0.001$ & 4.606 & $2.422-8.758$ & $<0.001$ \\
\hline Tumor size $(>3.6 / \leq 3.6)$ & 1.156 & $0.667-2.004$ & 0.605 & & & \\
\hline Neoadjuvant therapy $(+/-)$ & 2.286 & $1.227-4.092$ & 0.005 & 4.177 & $2.231-7.819$ & $<0.001$ \\
\hline Adjuvant therapy (+/-) & 2.348 & $1.205-4.576$ & 0.012 & 0.782 & $0.333-1.834$ & 0.572 \\
\hline CD (III, IV/I, II) & 1.273 & $0.731-2.215$ & 0.394 & & & \\
\hline
\end{tabular}

OR, odds ratio; Cl, confidence interval; ASA PS, American Society of Anesthesiologists physical status; BMl, body mass index; CEA, carcinoembryonic antigen; MIS, minimally invasive surgery; $C D$, Clavien-Dindo classification.

sults are consistent with our institute's previous study showing that LVI, PNI, and tumor budding are as strong predictors of colorectal cancer disease-free survival [20]. Preoperative perforation and receiving neoadjuvant therapy could be related to the severity of cancer stage affecting recurrence.

An objective stratification protocol was used to classify patient complications based on treatment modality rather than clinical manifestations. The extended CD classification system that standardizes adverse event terms in more detail was used [21]. This helped us overcome the limitation of retrospective data collection. However, the CD classification system stratifies surgical complications based on the invasiveness of the treatment modality. This grading system may not reflect the impact of a patient's systemic condition after surgery [8].

As a retrospective single center study, our study has a few limitations. Type II error may be present due to the relatively small number of patients. In our study, 57 patients underwent preoperative chemoradiotherapy, so combining of ypStage with pStage can cause bias. Another limitation is that our study did not consider patient comorbidities. Even if a patient had many comorbidities, the most invasive treatment grade was based on extended CD classification. This bias might underestimate the comorbidity effect on patients.

In conclusion, advanced cancer stage and open surgery were identified as risk factors for increasing surgery-related major complications after radical colorectal cancer surgery. However, 
the severity of postoperative complications did not affect the disease-free survival of patients with colorectal cancer.

\section{CONFLICT OF INTEREST}

No potential conflicts of interest relevant to this article are reported.

\section{REFERENCES}

1. Bray F, Ferlay J, Soerjomataram I, Siegel RL, Torre LA, Jemal A. Global cancer statistics 2018: GLOBOCAN estimates of incidence and mortality worldwide for 36 cancers in 185 countries. CA Cancer J Clin 2018;68:394-424.

2. Liang J, Fazio V, Lavery I, Remzi F, Hull T, Strong S, et al. Primacy of surgery for colorectal cancer. Br J Surg 2015;102:847-52.

3. Yamamoto S, Inomata M, Katayama H, Mizusawa J, Etoh T, Konishi F, et al. Short-term surgical outcomes from a randomized controlled trial to evaluate laparoscopic and open D3 dissection for stage II/III colon cancer: Japan Clinical Oncology Group Study JCOG 0404. Ann Surg 2014;260:23-30.

4. Clinical Outcomes of Surgical Therapy Study Group, Nelson H, Sargent DJ, Wieand HS, Fleshman J, Anvari M, et al. A comparison of laparoscopically assisted and open colectomy for colon cancer. N Engl J Med 2004;350:2050-9.

5. Veldkamp R, Kuhry E, Hop WC, Jeekel J, Kazemier G, Bonjer HJ, et al. Laparoscopic surgery versus open surgery for colon cancer: short-term outcomes of a randomised trial. Lancet Oncol 2005;6: 477-84.

6. Richards CH, Platt JJ, Anderson JH, McKee RF, Horgan PG, McMillan DC. The impact of perioperative risk, tumor pathology and surgical complications on disease recurrence following potentially curative resection of colorectal cancer. Ann Surg 2011; 254:83-9.

7. Aoyama T, Oba K, Honda M, Sadahiro S, Hamada C, Mayanagi S, et al. Impact of postoperative complications on the colorectal cancer survival and recurrence: analyses of pooled individual patients' data from three large phase III randomized trials. Cancer Med 2017;6:1573-80.

8. Mrak K, Eberl T, Laske A, Jagoditsch M, Fritz J, Tschmelitsch J. Impact of postoperative complications on long-term survival after resection for rectal cancer. Dis Colon Rectum 2013;56:20-8.

9. Odermatt M, Miskovic D, Flashman K, Khan J, Senapati A, O'Leary D, et al. Major postoperative complications following elective resection for colorectal cancer decrease long-term survival but not the time to recurrence. Colorectal Dis 2015;17:141-9.

10. Fagard K, Leonard S, Deschodt M, Devriendt E, Wolthuis A, Prenen $\mathrm{H}$, et al. The impact of frailty on postoperative outcomes in individuals aged 65 and over undergoing elective surgery for colorectal cancer: a systematic review. J Geriatr Oncol 2016;7:47991.

11. McSorley ST, Horgan PG, McMillan DC. The impact of the type and severity of postoperative complications on long-term outcomes following surgery for colorectal cancer: a systematic review and meta-analysis. Crit Rev Oncol Hematol 2016;97:168-77.

12. Breugom AJ, van Dongen DT, Bastiaannet E, Dekker FW, van der Geest LG, Liefers GJ, et al. Association between the most frequent complications after surgery for stage I-III colon cancer and shortterm survival, long-term survival, and recurrences. Ann Surg Oncol 2016;23:2858-65.

13. Abramovitch R, Marikovsky M, Meir G, Neeman M. Stimulation of tumour growth by wound-derived growth factors. Br J Cancer 1999;79:1392-8.

14. Mynster T, Christensen IJ, Moesgaard F, Nielsen HJ. Effects of the combination of blood transfusion and postoperative infectious complications on prognosis after surgery for colorectal cancer. Danish RANX05 Colorectal Cancer Study Group. Br J Surg 2000; 87:1553-62.

15. O'Dwyer PJ, Martin EW Jr. Viable intraluminal tumour cells and local/regional tumour growth in experimental colon cancer. Ann R Coll Surg Engl 1989;71:54-6.

16. Goldfarb Y, Sorski L, Benish M, Levi B, Melamed R, Ben-Eliyahu S. Improving postoperative immune status and resistance to cancer metastasis: a combined perioperative approach of immunostimulation and prevention of excessive surgical stress responses. Ann Surg 2011;253:798-810.

17. Dunn GP, Old LJ, Schreiber RD. The immunobiology of cancer immunosurveillance and immunoediting. Immunity 2004;21:13748.

18. Watt DG, McSorley ST, Park JH, Horgan PG, McMillan DC. A postoperative systemic inflammation score predicts short- and long-term outcomes in patients undergoing surgery for colorectal cancer. Ann Surg Oncol 2017;24:1100-9.

19. Slankamenac K, Slankamenac M, Schlegel A, Nocito A, Rickenbacher A, Clavien PA, et al. Impact of postoperative complications on readmission and long-term survival in patients following surgery for colorectal cancer. Int J Colorectal Dis 2017;32:805-11.

20. Huh JW, Lee WY, Shin JK, Park YA, Cho YB, Kim HC, et al. A novel histologic grading system based on lymphovascular invasion, perineural invasion, and tumor budding in colorectal cancer. J Cancer Res Clin Oncol 2019;145:471-7.

21. Katayama H, Kurokawa Y, Nakamura K, Ito H, Kanemitsu Y, Masuda N, et al. Extended Clavien-Dindo classification of surgical complications: Japan Clinical Oncology Group postoperative complications criteria. Surg Today 2016;46:668-85.

22. Dindo D, Demartines N, Clavien PA. Classification of surgical complications: a new proposal with evaluation in a cohort of 6336 patients and results of a survey. Ann Surg 2004;240:205-13.

23. Sanchez-Velazquez P, Pera M, Jimenez-Toscano M, Mayol X, Roges X, Lorente $\mathrm{L}$, et al. Postoperative intra-abdominal infection is an independent prognostic factor of disease-free survival and disease-specific survival in patients with stage II colon cancer. Clin Transl Oncol 2018;20:1321-8.

24. Ma Y, Yang Z, Qin H, Wang Y. A meta-analysis of laparoscopy 


\section{Coloproctology Chang Kyu Oh, et al.}

compared with open colorectal resection for colorectal cancer. Med Oncol 2011;28:925-33.

25. Huh JW, Kim HC, Kim SH, Park YA, Cho YB, Yun SH, et al. Tumor regression grade as a clinically useful outcome predictor in patients with rectal cancer after preoperative chemoradiotherapy. Surgery 2019;165:579-85.

26. Shimada H, Fukagawa T, Haga Y, Oba K. Does postoperative morbidity worsen the oncological outcome after radical surgery for gastrointestinal cancers? A systematic review of the literature. Ann Gastroenterol Surg 2017;1:11-23.

27. Timmers TK, Verhofstad MH, Moons KG, Leenen LP. Long-term survival after surgical intensive care unit admission: fifty percent die within 10 years. Ann Surg 2011;253:151-7.

28. Park JS, Huh JW, Park YA, Cho YB, Yun SH, Kim HC, et al. Risk factors of anastomotic leakage and long-term survival after colorectal surgery. Medicine (Baltimore) 2016;95:e2890.

29. Cienfuegos JA, Baixauli J, Beorlegui C, Ortega PM, Granero L, Zozaya $\mathrm{G}$, et al. The impact of major postoperative complications on long-term outcomes following curative resection of colon cancer. Int J Surg 2018;52:303-8.

30. de Haas RJ, Wicherts DA, Andreani P, Pascal G, Saliba F, Ichai P, et al. Impact of expanding criteria for resectability of colorectal metastases on short- and long-term outcomes after hepatic resection. Ann Surg 2011;253:1069-79. 